\title{
Design Flexibility of Ultra-High Efficiency 4-Junction Inverted Metamorphic Solar Cells
}

\author{
Ryan M. France , John F. Geisz , Iván García , Myles A. Steiner ${ }^{1}$ William E. McMahon , Daniel J. \\ Friedman , Tom E. Moriarty , Carl Osterwald , J. Scott Ward , Anna Duda , Michelle Young , and Waldo J. \\ Olavarria
}

\begin{abstract}
We present recent improvements to the 4-junction inverted metamorphic solar cell. The device now includes a (Ga)InAsP buffer that transitions to lattice constants greater than InP, which allows access to GaInAs subcells with bandgaps $<0.74 \mathrm{eV}$ and an additional $2 \mathrm{~mA} / \mathrm{cm}^{2}$ of bottom junction photocurrent at AM1.5D. However, the optimal design depends on the spectrum and operating temperature. We show how the device flexibility can be used to fine-tune the design for various spectra in order to maximize energy yield for a given operating condition. 1-sun devices achieve $35.3 \%$ efficiency under the AM0 spectrum and $37.8 \%$ efficiency under the global spectrum at $25^{\circ} \mathrm{C}$. Concentrator devices achieve $45.7 \%$ peak efficiency under $234 x$ the direct spectrum and maintain over $45 \%$ efficiency at $700 x$ at $25^{\circ} \mathrm{C}$. Other device improvements include a 4-layer antireflection coating with low power loss, and reduced series resistance.
\end{abstract}

Index Terms - 4-junction solar cells, inverted metamorphic multijunction

\section{INTRODUCTION}

The 4-junction inverted metamorphic (4J-IMM) solar cell has been shown to be capable of achieving extremely high efficiency despite the use of lattice-mismatched subcells [1]. Lattice-matched high-bandgap GaInP and GaAs subcells are grown first on a GaAs substate. Then, compositionally graded buffers are used to transition the lattice constant to two GaInAs subcells with tunable bandgaps. By using an optimized structure and growth conditions in the graded buffers, the dislocation density in the mismatched subcells is kept low. Carrier collection in the mismatched subcells is nearly perfect, and voltages can be almost as high as in their lattice-matched counterparts. With this bandgap flexibility, the device can be fine-tuned for different spectra. Here, we show recent improvements to the $4 \mathrm{~J}-\mathrm{IMM}$, and demonstrate high efficiencies under a variety of spectra achieved by small changes in the device design, allowing the device to be optimized for a particular location and operating condition.

\section{DEVICE DEVELOPMENT}

The device optimization of the 4-junction inverted metamorphic solar cell can be an extremely daunting task that depends on the nonradiative recombination and diffusion length of all junctions at all potential bandgaps, the antireflection coating, series resistances, and the expected operating spectra, concentration and temperature. The portion of the solar spectra that is potentially useful for 4 -junction terrestrial cells extends to the water absorption edge at approximately $0.70 \mathrm{eV}$. Previously [1], we showed a 4J-IMM using a compositionally graded buffer that transitions the lattice-constant to InP, creating a bottom GaInAs cell with $0.74 \mathrm{eV}$ at $25^{\circ} \mathrm{C}$. To absorb light between 0.74 and $0.70 \mathrm{eV}$, for a potential $2 \mathrm{~mA} / \mathrm{cm}^{2}$ increase in photocurrent, a graded buffer beyond InP is required. In this work, we have developed a (Ga)InAsP buffer that allows GaInAs with a bandgap $<0.74 \mathrm{eV}$, improving the bandgap tunability of the device.

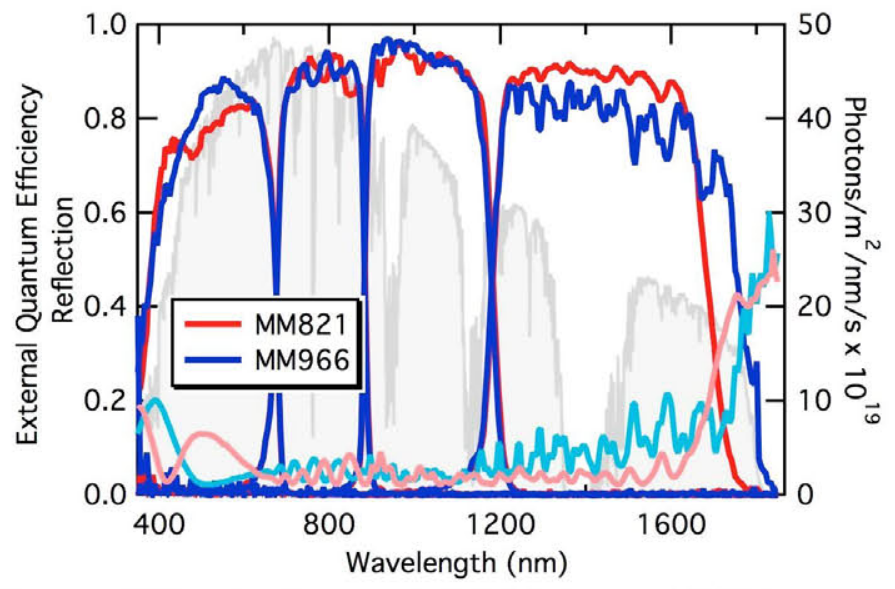

Fig. 1. EQE and reflection of 4J-IMM devices. MM821 uses a $0.74-\mathrm{eV}$ bottom junction, with $3.8 \%$ misfit, and MM966 uses a 0.70 $\mathrm{eV}$ bottom junction, with $4.1 \%$ misfit. MM966 has an ARC that transmits more short-wavelength light in order to raise the multijunction current.

Figure 1 shows the EQE and reflection of two devices utilizing different bottom cells. Each device utilizes a 4-layer anti-reflection coating specifically designed to maximize the current for the particular multijunction device by tuning the reflectance. Device MM966 incorporates a lower bandgap bottom cell, $0.70 \mathrm{eV}$, which increases the absorption range of the multijunction cell. To appropriately distribute the incident light, device MM966 uses an ARC whose reflection is 
weighted more towards the long-wavelength range rather than the short-wavelength range. These changes increase total absorption and redistribute photons between the junctions for an increase in the photocurrent of the top cell by $0.4 \mathrm{~mA} / \mathrm{cm}^{2}$ and the bottom cell by $0.6 \mathrm{~mA} / \mathrm{cm}^{2}$ with respect to device MM821, as determined from the integral of the product of the EQE and direct spectrum. The one-sun $\mathrm{J}_{\text {sc }}$ improves by 0.5 $\mathrm{mA} / \mathrm{cm}^{2}$ and absolute efficiency (NREL certified) is boosted by $1.8 \%$, as measured with a spectrally adjustable one-sun simulator.

a)

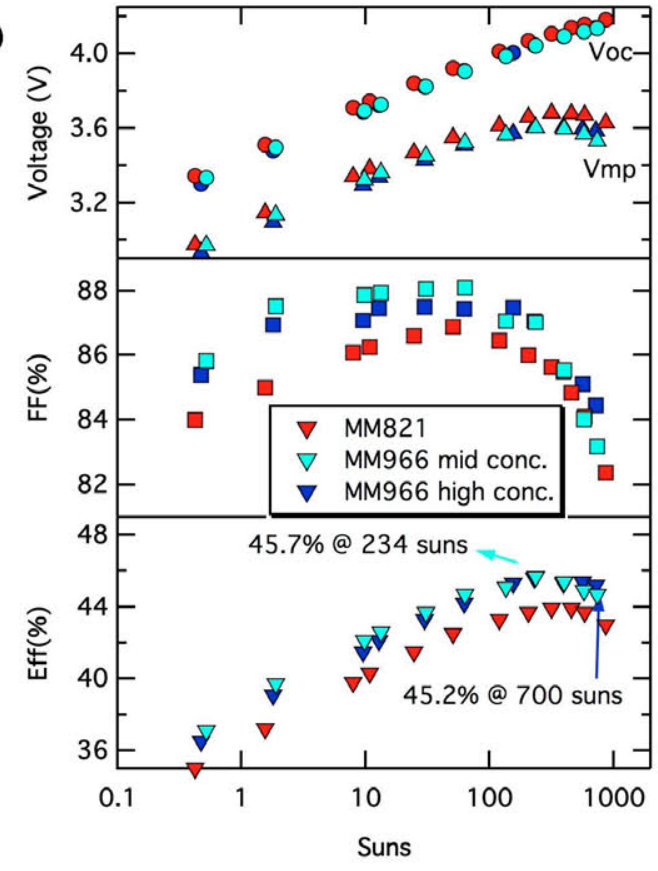

b)

NREL

GaInP/GaAs/GaInAs/GaInAs Quad Cell

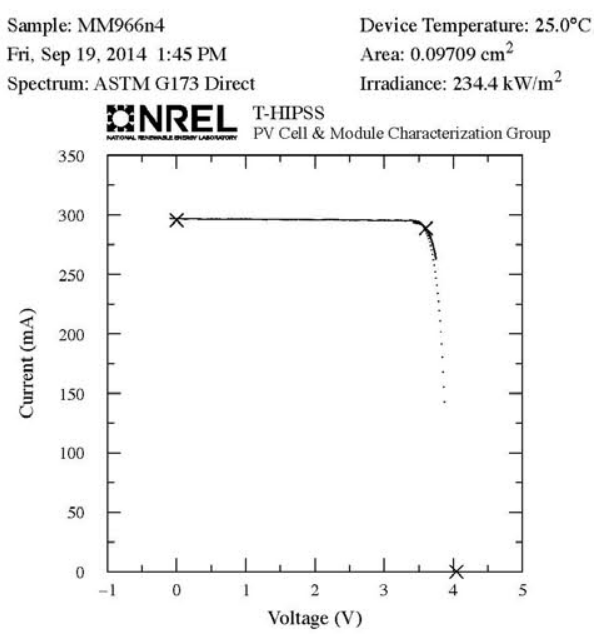

$$
\begin{array}{ll}
\mathrm{V}_{\mathrm{oc}}=4.043 \mathrm{~V} & \mathrm{~V}_{\max }=3.603 \mathrm{~V} \\
\mathrm{I}_{\mathrm{sc}}=0.2955 \mathrm{~A} & \mathrm{I}_{\max }=0.2885 \mathrm{~A} \\
\text { Fill Factor }=87.03 \% & \mathrm{P}_{\max }=1.040 \mathrm{~W} \\
\text { Efficiency }=45.7 \pm 2.3 \% &
\end{array}
$$

Fig. 2. (a) Performance of 4J-IMM solar cells under concentrated light (NREL certified), taken using a T-HIPSS flash simulator. (b) MM966 achieves $45.7 \pm 2.3 \%$ efficiency at 234 suns by having broadband absorption and low series resistance losses.

Front grids are designed for minimal power loss at both mid and high concentration for the measured top cell sheet resistance and contact resistance. The GaInP sheet resistance of these devices is low, $115 \mathrm{Ohm} / \mathrm{sq}$, due to the thick, moderately doped emitter of the rear-heterojunction GaInP subcell [2]. Figure 2 shows the concentration results (NREL certified) of device MM966 with two grid spacings, designed for mid and high concentration levels. The grid spacings are $300 \mu \mathrm{m}$ and $200 \mu \mathrm{m}$, respectively. The peak efficiency is 45.7 $+/-2.3 \%$ under $234 \mathrm{x}$ the direct spectrum, and $45.2+/-2.3 \%$ at 700x. The measurements under concentrated light were taken with an adjustable flash simulator (tunable-high intensity pulsed solar simulator, or T-HIPSS), which was tuned using photocurrent ratio balancing [3]. Photocurrent ratios $\left(\mathrm{R}_{11}, \mathrm{R}_{21}\right.$, $\left.R_{31}, R_{41}\right)=(1,1.01,1.01,0.96)$ for this cell, indicating that an appropriate spectrum was achieved with the flash simulator.

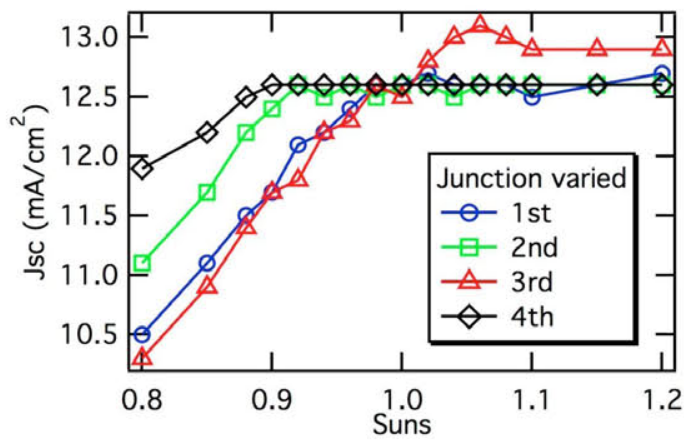

Fig. 3. Varied irradiance measurement on a 4-junction device. The Jsc of the multijunction device is measured as the irradiance to each junction is varied. The irradiance to all the other junctions is adjusted to one-sun.

Further device optimization is in progress. To optimize the bandgap combination for a given spectrum, the subcell photocurrents must be measured. For lowest error, we vary the irradiance on an adjustable one-sun simulator to determine the subcell photocurrents rather than integrating the EQE. Figure 3 shows an example of a varied irradiance measurement on device MN187 using a one-sun multisource simulator (OSMSS) with nine controllable spectral zones. The spectrum is initially set to one-sun, and then the irradiance to each junction is systematically varied as the total multijunction current is measured. The measurement shows that the subcell photocurrents are not equal, and that $3^{\text {rd }}$ subcell limits the multijunction current. To optimize this multijunction device, the photocurrent of the $3^{\text {rd }}$ subcell should be raised, either by increasing the bandgap or decreasing the thickness of the $2^{\text {nd }}$ subcell, or lowering the bandgap of the $3^{\text {rd }}$ subcell. 


\section{DESIGNS}

The optimal bandgap combination and subcell thicknesses depend on the spectrum and operating temperature, and the actual subcell performance. The design flexibility of the $4 \mathbf{J}$ IMM allows fine-tuning the device for different operating conditions, and maximizing the yearly energy yield of the device [4-5]. To illustrate this design flexibility, we designed multijunction solar cells for the direct, global, and AMO spectra. The thickness of the lattice-matched subcells, and bandgaps of the lattice-mismatched subcells were adjusted in order to optimize the current. With a fixed $2^{\text {nd }}$ subcell bandgap, device changes can be simply explained by noting the ratio (photons with energy $>1.42 \mathrm{eV}$ )/(photons with energy $>0.69 \mathrm{eV}$ ) for the direct, global, and AMO spectra: $0.517,0.534$, and 0.492 , respectively, given an EQE representative of these devices. For the global spectrum, we use a bottom subcell with $0.70 \mathrm{eV}$ to maximize current for applications at $25^{\circ} \mathrm{C}$. The $2^{\text {nd }}$ subcell is thinned to allow additional light to access the bottom subcells. For the AMO spectrum, the top cell is thinned to allow light to the GaAs cell. The bottom subcells receive excess photocurrent so higher bandgaps are used to raise the voltage. The efficiencies under the global and AMO spectra are 37.8\% (MN385), and $35.3 \%$ (MM987), respectively (NREL certified).

Table I: 4J-IMMs with different designs. The thicknesses of the top two subcells, and bandgaps of the bottom two subcells, are adjusted to optimize the photocurrent distribution. All measurements at 1-sun irradiance

\begin{tabular}{|l|l|l|l|}
\hline & Direct & Global & AM0 \\
\hline $1^{\text {st }} \mathrm{J}$. thickness $(\mathrm{nm})$ & 800 & 800 & 550 \\
\hline $2^{\text {nd }} \mathrm{J}$. thickness $(\mathrm{nm})$ & 3000 & 2000 & 3000 \\
\hline $3^{\text {rd }} \mathrm{J}$. bandgap $\left(\mathrm{E}_{\mathrm{g}}\right)$ & 1.04 & 1.02 & 1.04 \\
\hline $4^{\text {th }} \mathrm{J}$. bandgap $\left(\mathrm{E}_{\mathrm{g}}\right)$ & 0.72 & 0.70 & 0.74 \\
\hline Jsc $\left(\mathrm{mA} / \mathrm{cm}^{2}\right)$ & 12.5 & 13.1 & 15.9 \\
\hline FF $(\%)$ & 85 & 84 & 84 \\
\hline Voc $(\mathrm{V})$ & 3.44 & 3.45 & 3.53 \\
\hline 1 -sun efficiency $(\%)$ & 36.6 & 37.8 & 35.3 \\
\hline
\end{tabular}

For terrestrial spectra, the expected operating temperature impacts the optimal bottom cell bandgap, in order to maximize voltage while collecting the light above the $0.70-\mathrm{eV}$ absorption valley. For the direct spectrum, we use $0.72 \mathrm{eV}$ for the bottom cell for robust performance at elevated operating temperature. A $0.72-\mathrm{eV}$ bottom junction will not be affected by the absorption gap in the direct spectrum until temperatures of $350 \mathrm{~K}$ and above. Device MN187, a concentrator device with $4 \%$ shadowing, achieves $36.6 \%$ efficiency at $25^{\circ} \mathrm{C}$ (NREL certified). Further analyses of the limitations of these designs, and routes to higher efficiency will be presented.

\section{CONCLUSION}

We have improved the 4-junction IMM by developing a $0.70-\mathrm{eV}$ bandgap bottom cell, which raises the current, and reducing series resistance, which lowers power loss at concentration. A concentrator device with of $45.7+/-2.3 \%$ peak efficiency at 234 suns is demonstrated. 4-junction solar cells are specifically designed for the direct, global and AMO spectra, demonstrating the capability to fine-tune the device design for optimal performance under different operating conditions.

\section{REFERENCES}

[1] R.M. France et al., "Quadruple-Junction Inverted Metamorphic Devices," IEEE J. Photovoltaics, vol. 5, pp. 432-437, 2015.

[2] J.F. Geisz, M.A. Steiner, I. Garcia, S.R. Kurtz, and D.J. Friedman, "Enhanced external radiative efficiency for $20.8 \%$ efficienct single-junction GaInP solar cells," Appl. Phys Lett., vol. 103, pp. 041118 1-5, 2013.

[3] T. Moriarty, J. Jablonski, and K. Emery, "Algorithm for building a simu- lator spectrum for NREL one-sun multi-source simulator," in Proc. 38th Photovoltaic Spec. Conf., Austin, TX, USA, 2012.

[4] I. Garcia, W.E. McMahon, M.A. Steiner, J.F. Geisz, A. Habte, and D.J. Friedman, "Optimization of Multijunction Solar Cells Through Indoor Energy Yield Measurements", IEEE J. Photovoltaics, vol. 5, pp. 438-445, 2015.

[5] I. Garcia, W.E. McMahon, A. Habte, J.F. Geisz, M.A. Steiner, M. Sengupta, and D.J. Friedman, "Field Spectra Binning for Energy Yield Calculations and Solar Cell Design", submitted to $42^{\text {nd }}$ IEEE PVSC, 2015. 